\title{
Comunicación
}

\section{Primeros casos de infección canina con Hepatozoon canis en la ciudad de Cúcuta, Colombia}

\author{
First cases of canine infection with Hepatozoon canis in the city of Cúcuta, \\ Colombia
}

\author{
Daniel Leonardo Cala Delgado ${ }^{1,3,5}$, Arly Katerine Noguera Gaona ${ }^{2}$, Norquis Caled \\ Álvarez Rubio ${ }^{3}$, Jefferson Yunis Aguinaga ${ }^{4}$
}

\section{Resumen}

Se describen dos casos de caninos infectados con Hepatozoon canis en la ciudad de Cúcuta, Colombia. La primera paciente de 18 meses de edad llegó a consulta con vómitos, anorexia y pérdida progresiva de peso. En el segundo caso se pretendía realizar ovariohisterectomía preventiva, pero en los exámenes clínicos prequirúrgicos presentó anemia. Para los dos casos, el diagnóstico fue mediante extendido sanguíneo, observado a 100X. Para el segundo caso, el diagnóstico se confirmó con la prueba de PCR. El tratamiento instaurado fue sintomático, usando fármacos como oxitetraciclina y doxiciclina. Este es el primer reporte de hepatozoonosis en la ciudad de Cúcuta.

Palabras clave: emergente; frotis; ectoparásitos; protozoario; PCR

\section{Abstract}

Two cases of canines infected with Hepatozoon canis are described in the city of Cúcuta, Colombia. The first patient of 18 months of age came to consultation with vomiting, anorexia, and progressive weight loss. In the second case, it was intended to perform

\footnotetext{
${ }^{1}$ Grupo de Investigación Los Araucos, Semillero de Investigación Cabalgando, Universidad Cooperativa de Colombia, sede Arauca. Arauca, Colombia

${ }^{2}$ Clínica Veterinaria Quinta Vélez, Cúcuta, Norte de Santander, Colombia

${ }^{3}$ Facultad de Medicina Veterinaria y Zootecnia, Universidad Cooperativa de Colombia, sede Arauca, Colombia

${ }^{4}$ Facultad de Ciencias Agrarias y Veterinarias, Universidad Estadual Paulista, Jaboticabal, Sao Paulo, Brasil

${ }^{5}$ E-mail: daniel.cala@campusucc.edu.co
}

Recibido: 9 de febrero de 2018

Aceptado para publicación: 19 de julio de 2018 
preventive ovariohysterectomy, but the patient presented anemia in the pre-surgical clinical tests. For both cases, the diagnosis was by blood smear, observed at 100X. For the second case the diagnose was confirmed by PCR. The treatment established was symptomatic, using drugs such as oxytetracycline and doxycycline. This is the first report of hepatozoonosis in the city of Cúcuta.

Key words: emergent; smear; ectoparasites; protozoa; PCR

\section{INTRODUCCIÓN}

La hepatozoonosis es producida por un protozoario del género Hepatozoon, conformado por cerca de 300 especies ampliamente distribuidas a nivel mundial (Sumrandee $e t$ al., 2015). La enfermedad se reporta en reptiles (Maia et al., 2014), aves (Merino et al., 2014) y mamíferos (Meneses et al., 2016). El Hepatozoon se encuentra en hospederos invertebrados hematófagos como garrapatas, ácaros, mosquitos, pulgas, piojos y moscas (Smith, 1996; Baneth et al., 2001; Van et al., 2015). La transmisión se presenta cuando el hospedador vertebrado intermediario ingiere el hospedador invertebrado definitivo (Greene, 2012). No se encuentran reportes de transmisión por medio de la saliva de los invertebrados hematófagos, como ocurre en otras enfermedades hemoparasitarias (Allen et al., 2008).

La ingestión de invertebrados infectados con ooquistes esporulados por parte del huésped intermediario genera la liberación de esporozoítos en el tracto gastrointestinal. Estos atraviesan la pared del intestino y son transportados por el sistema circulatorio hasta los órganos diana (hígado, bazo y médula ósea), donde ocurre la fase de merogonia dando como resultado a merozoítos. Estos penetran a los neutrófilos y se transforman en gamontes que aparecen en la sangre periférica (Gavazza et al., 2003). El ciclo se cierra cuando los hospederos invertebrados succionan sangre infectada del hospedador intermedio (Baneth et al., 2007).
No todos los animales diagnosticados con hepatozoonosis presentan manifestaciones clínicas (Baneth et al., 2013); sin embargo, cuando los organismos infectados se encuentran inmunosuprimidos pueden mostrar respuestas clínicas caracterizadas por miosistis crónica, debilidad y muerte (Mateus et al., 2007). La hepatozoonosis en caninos produce anemia, fiebre, letargia, caquexia y pérdida progresiva de peso (Baneth et al., 2003). Otras lesiones asociadas a la hepatozoonosis son uveítis, glaucoma, osteopatía hipertrófica, poliartritis y linfadenitis (Headley et al., 2005; Acevedo et al., 2009; Iveli et al., 2015).

En América se han presentado reportes de H. canis en Brasil (Ramos et al., 2015), Estados Unidos de Norteamérica (Allen et al., 2008), Venezuela (Gómez et al., 2015), Paraguay (Tintel, 2016) y Argentina (Ruiz et al., 2013). El primer reporte en Colombia se hizo en 2004 en un canino macho Pitbull diagnosticado por medio de extendido sanguíneo en el laboratorio del Centro Médico Quirúrgico Veterinario de la Universidad Cooperativa de Colombia (CMQV-UCC), sede Bucaramanga (Arcila et al., 2005).

La hepatozoonosis en Colombia fue considerada por Castellanos (2008) como enfermedad emergente, luego de haberse documentado la ocurrencia de cinco casos entre 2004 y 2007 en la ciudad de Bucaramanga (Mateus et al., 2007). En el departamento de Antioquia fue expuesto otro caso de $H$. canis en 2009, donde el paciente presentó uveítis y glaucoma, lesiones asociadas a la infección 
por este patógeno (Acevedo et al., 2009). Por otro lado, no ha sido reportada la presencia de hepatozoonosis en caninos de otros departamentos de Colombia.

En el presente artículo se describen los dos primeros reportes de Hepatozoon canis en caninos de la ciudad de Cúcuta, departamento de Norte de Santander, Colombia.

\section{Casos Clínicos}

\section{Primer Caso}

\section{Anamnesis}

El 1 de febrero de 2016 fue atendido en la Clínica Veterinaria «Quinta Vélez», de la ciudad de Cúcuta, Norte de Santander, un canino hembra Schnauzer, de 1.5 años, $7 \mathrm{~kg}$ de peso. El propietario reporta que la mascota ha vomitado durante tres días seguidos, ha perdido peso, no realiza sus actividades con normalidad y presenta anorexia. La paciente cuenta con carné de vacunación y desparasitación actualizado.

\section{Hallazgos al examen clínico}

Paciente de carácter dócil, en estado caquéctico, condición corporal considerada como muy delgada ( 3 en una escala de 1 a 9) (Freeman et al., 2011). A la palpación se encontró linfadenitis submandibular bilateral, dolor en el miembro posterior izquierdo, temperatura de $40.4{ }^{\circ} \mathrm{C}$, mucosa ocular y gingival rosa pálido, tiempo de llenado capilar de $4 \mathrm{~s}$, frecuencia cardiaca $110 \mathrm{lpm}$, frecuencia respiratoria de 22 por minuto y presencia de gran cantidad de garrapatas Rhipicephalus sanguineus.

El diagnóstico presuntivo fue de enfermedades causadas por patógenos hematófagos como Ehrlichia sp y Anaplasma sp. No obstante, Hepatozoon canis fue consi- derado como posibilidad, aunque de menor prioridad, dado que aún no se habían realizado reportes en Cúcuta.

\section{Ayudas diagnósticas}

Se colectó sangre de la vena cefálica en tubo con EDTA para la realización de hemograma y en tubo sin anticoagulante para obtener suero sanguíneo y evaluar niveles de alanina transaminasa y creatinina. Se evidenció ligera anemia (33\%; referencia: $35-45 \%$ ) $\mathrm{y}$ hemoglobina en el rango inferior, $(11 \mathrm{~g} / \mathrm{dl}$ : referencia: 11.6-15 g/dl). El recuento leucocitario reportó un aumento (30 400/ml; referencia: $7000-12000 / \mathrm{ml})$, neutrofilia $(96 \%$, referencia: $50-70 \%$ ). Los valores de alanina transaminasa y creatinina fueron normales (valores de referencia: Pedroso et al., 2010).

Se empleó la prueba de $\mathrm{SNAP}^{\circledR} 4 \mathrm{Dx}^{\circledR}$ Plus (Laboratorios IDEXX) para descartar enfermedades transmitidas por vectores (Dirofilaria immitis, Anaplasma phagocytophilum, A. platys, Borrelia burgdorferi, Ehrlichia canis y Ehrlichia ewingii) con resultado negativo. Con base a esto, se hicieron frotis sanguíneos con tinción hemacolor en el laboratorio de diagnóstico veterinario Labovet, pudiéndose constatar la presencia de un cuerpo ovalado en los glóbulos blancos; un neutrófilo con gametocito de Hepatozoon sp (Figura 1). No obstante, no se pudo confirmar por medio del PCR.

\section{Tratamiento}

El tratamiento inicial fue para mejorar la condición corporal y estabilizar el paciente. Luego del diagnóstico de infección por Hepatozoon sp se procedió a controlar la presencia del parásito durante nueve días de hospitalización (Cuadro 1) y posterior a ella (Cuadro 2). Se programó un control a los ocho días del tratamiento inicial observando evolución favorable. Los valores hemáticos se encontraron en los rangos normales para la especie en los controles posteriores. 


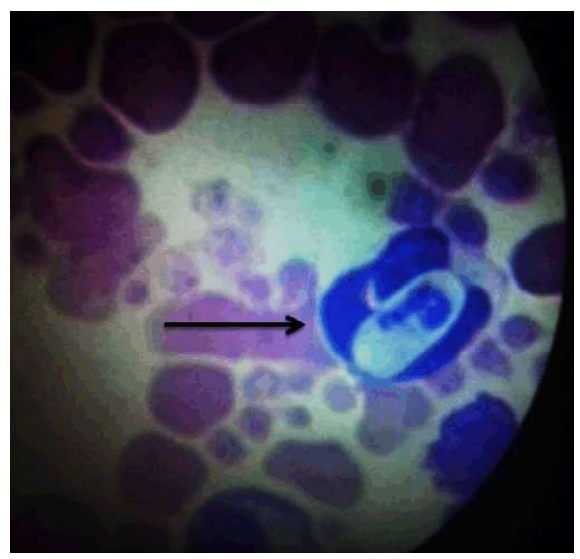

Figura 1. Frotis sanguíneo de perra. Se observa neutrófilo parasitado con gametocito de Hepatozoon sp (Flecha) Tinción Hemacolor ${ }^{\circledR}$ x 100

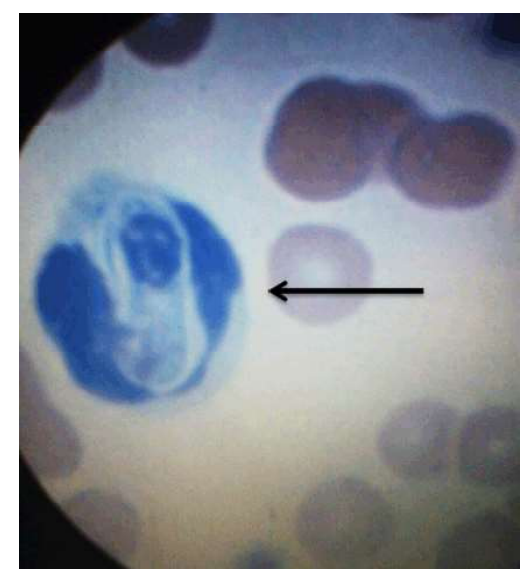

Figura 2. Frotis sanguíneo de perra. Se observa neutrófilo parasitado con gametocito de Hepatozoon sp (Flecha) Tinción Hemacolor ${ }^{\circledR}$ x 100

\section{Segundo Caso}

\section{Anamnesis}

Un canino hembra, de raza Lobo Siberiano, de 19 meses de edad, con peso de $17 \mathrm{~kg}$ fue llevado a la Clínica Veterinaria Quinta Vélez el 12 de enero de 2017 para que se le realizara una ovariohisterectomía $(\mathrm{OVH})$ preventiva. Las vacunas y despara- sitación se encontraban al día. La paciente fue tratada contra hemoparásitos dos meses atrás y presentó epistaxis. Aparentemente, la paciente se encontraba sana al ingresar a la clínica.

\section{Hallazgos al examen clínico}

Paciente inquieto, condición corporal de 5 (escala de 1 a 9) (Freeman et al., 2011), mucosa oral color rosa, tiempo de llenado capilar $3 \mathrm{~s}$, frecuencia cardiaca 130 $\mathrm{lpm}$, temperatura corporal $38.5^{\circ} \mathrm{C}$. La frecuencia respiratoria no se pudo evaluar dada la agitación del paciente.

\section{Ayudas diagnósticas}

Se realizó un cuadro hemático prequirúrgico encontrando anemia (hematocrito 25\%, referencia: $35-45 \%$ ), hemoglobinemia ( $8.3 \mathrm{~g} / \mathrm{dl}$, referencia: $11.6-$ $15 \mathrm{~g} / \mathrm{dl})$, leucocitosis $(16000 / \mathrm{ml}$, referencia: $7000-12000 / \mathrm{ml})$ y neutrofilia $(82 \%$, referencia: $50-70 \%$ ). Las proteínas totales, plaquetas y creatinina estaban en rangos adecuados para la especie y la edad. En el frotis sanguíneo se observaron cuerpos de inclusión en algunos glóbulos blancos (Figura 2). (valores de referencia: Pedroso et al., 2010).

Mediante la metodología descrita por Lata et al. (2009), se confirma que las muestras fueron negativas para Anaplasma sp y Ehrlichia sp, pero positivas para Hepatozoon canis y Babesia sp. La prueba cuenta con $99.9 \%$ de sensibilidad y especificidad, según el laboratorio de genética animal Mascolab en la ciudad de Cúcuta, Norte de Santander, Colombia.

\section{Tratamiento}

El paciente fue hospitalizado por 10 días, administrándosele fluidos endovenosos $(0.9 \%$ $\mathrm{NaCl}$ ), oxitetraciclina (4 mg/kg IV c/12 h), 
Cuadro 1. Tratamiento instaurado durante la hospitalización en paciente canino diagnosticado con Hepatozoon sp

\begin{tabular}{lcccc}
\hline Medicamento & $\begin{array}{c}\text { Dosis } \\
(\mathrm{mg} / \mathrm{kg})\end{array}$ & Vía & $\begin{array}{c}\text { Intervalo } \\
\text { (horas) }\end{array}$ & $\begin{array}{c}\text { Duración } \\
\text { (días) }\end{array}$ \\
\hline Ondansetrón & 0.5 & IV & 12 & 3 \\
Omeprazol & 0.5 & IV & 12 & 3 \\
Dexametasona & 0.5 & IV & 12 & 1 \\
Oxitetraciclina & 4 & IV & 12 & 7 \\
Toltrazuril $^{1}$ & 20 & PO & 24 & - \\
Imizol $^{\circledR 1}$ & 6 & SC & - & - \\
\hline
\end{tabular}

${ }^{1}$ Se administró al segundo día de hospitalización

Cuadro 2. Tratamiento recomendado post-hospitalización en paciente canino diagnosticado con Hepatozoon sp

\begin{tabular}{|c|c|c|c|c|}
\hline Medicamento & Dosis & Vía & $\begin{array}{c}\text { Intervalo } \\
\text { (horas) }\end{array}$ & $\begin{array}{c}\text { Duración } \\
\text { (días) }\end{array}$ \\
\hline Diciclin $100^{\circledR 1}$ & $1 / 2$ tableta & PO & 12 & 15 \\
\hline Toltrazuril & $2.4 \mathrm{ml}$ & $\mathrm{PO}$ & 24 & 7 \\
\hline Hemolitan $^{\circledR}$ & $1 \mathrm{ml}$ & PO & 12 & 20 \\
\hline $\operatorname{Imizol}^{\circledR 2}$ & $6 \mathrm{mg} / \mathrm{kg}$ & $\mathrm{SC}$ & - & $2^{\text {da }}$ dosis \\
\hline Hill's ${ }^{\circledR}$ Prescription Diet ${ }^{\circledR} a / d^{\circledR}$ & $65 \mathrm{~g}$ & $\mathrm{PO}$ & 6 & 15 \\
\hline
\end{tabular}

${ }^{1}$ Después de la alimentación

${ }^{2}$ Control en la clínica

doxiciclina $(5 \mathrm{mg} / \mathrm{kg} \mathrm{PO} \mathrm{c} / 12 \mathrm{~h})$, Glicopan ${ }^{\circledR} \mathrm{Pet}$ $(0.5 \mathrm{ml} / \mathrm{kg} \mathrm{PO} \mathrm{c} / 12 \mathrm{~h})$. Los resultados del cuadro hemático de control a los ocho días de instaurado el tratamiento mostraron resultados normales para la especie y edad.

\section{Discusión}

Estos casos constituyen los primeros reportes de hepatozoonosis en caninos de la ciudad de Cúcuta, Colombia. El diagnóstico de Hepatozoon canis en muchos casos es accidental y se encuentra cuando los pacientes son llevados a las clínicas veterinarias por otros motivos (de Fonseca et al., 2016), al igual que el segundo caso del presente reporte.

La sintomatología clínica que mostraban los pacientes de estos casos clínicos fue muy similar a la descrito por Acevedo et al. (2009) en un canino de raza pastor alemán, con pérdida progresiva de peso, anorexia, decaimiento, dolor, temperatura de $39.5^{\circ} \mathrm{C}$; aunque tenía, además, uveítis y glaucoma asociados a Hepatozoon canis. La literatura científica 
documenta infecciones concomitantes de hepatozoonosis con Parvovirus (Hervas et al., 1995), Ehrlichia canis (Baneth et al., 2013), toxoplasmosis (Harmelin et al., 1992) y leishmaniasis (Rioux et al., 1964). En el segundo caso de este reporte, el paciente presentó infección concomitante con Babesia.

A pesar de la dificultad reportada por Mateus et al. (2007) de diagnosticar esta enfermedad por medio de extendidos sanguíneos observados en el microscopio a 100X, donde la probabilidad de encontrar células afectadas en 1-2 de 1000 leucocitos es baja, en estos dos casos la principal herramienta de diagnóstico fue el frotis sanguíneo. Se observaron los gametocitos, con una coloración azul brillante, de forma alargada, rectangulares, midiendo en promedio $11 \mu \mathrm{m}$ de largo y $5.2 \mu \mathrm{m}$ de ancho, tal y como lo describe Eljadar et al. (2013).

El porcentaje de positividad en los extendidos de sangre periférica no alcanza el 5\% (Castellanos, 2008), por lo cual se deben integrar otras ayudas diagnósticas. Las ecografías y radiografías permiten evidenciar alteraciones óseas, incremento de tamaño de órganos diana como hígado, bazo y ganglios (Adagio et al., 2014). La biopsia de músculo y parénquima de diferentes tejidos muestran la presencia de esquizontes y quistes del parásito (Restrepo, 2013). También se pueden evidenciar merontes maduros de Hepatozoon spp con merozoítos en su interior, en órganos como el bazo, causando esplenitis (Mejía y Acevedo, 2016).

Mateus et al. (2007) indica como tratamiento aceturato de diminaceno $(3.5 \mathrm{mg} / \mathrm{kg}$ IM, dosis única), dipropionato de imidocarb $(5 \mathrm{mg} / \mathrm{kg} \mathrm{SC}$, dosis única o repetir a los 14 días), así como combinaciones de Pirimitamina-Clindamicina $(0.25-10 \mathrm{mg} / \mathrm{kg}$, respectivamente. Así mismo, se utiliza toltrazuril asociado con dipropionato de imidocarb (Tintel, 2016). Por otro lado, Pasa et al. (2011) no encontraron diferencias entre el uso individual del dipropionato de imidocarb y la terapia asociativa con toltrazuril. Tratamientos experimentales con clindamicina demuestran reducción del porcentaje de animales infectados, mas no se logra una completa eliminación del parásito (de Tommasi et al., 2014). La espiramicina suministrada de forma oral en dosis de 23 $\mathrm{mg} / \mathrm{kg} \mathrm{c} / 24 \mathrm{~h}$ durante 10 días ayuda en la reducción de la parasitemia, pero no elimina totalmente el $H$. canis (Guendulain et al., 2017).

La hepatozoonosis es considerada una enfermedad emergente en Colombia (Castellanos, 2008). El ambiente tropical del país proporciona condiciones propicias para la proliferación de los vectores que transmiten la enfermedad, tales como $R$. sanguineus (Tintel, 2016) y R. turanicus (Giannelli et al., 2017). La ciudad de Cúcuta se caracteriza por tener climas adecuados para los transmisores de hemoparásitos, tales como Ehrlichia canis y Babesia; sin embargo, estos dos casos serían los primeros reportes de infección parasitaria en caninos por Hepatozoon sp, aunque se sospecha de la ocurrencia de casos previos, que por cuestiones económicas no se han diagnosticado de forma efectiva o por la baja probabilidad de observar el parásito por medio de frotis sanguíneos. La mejor forma de evitar estas enfermedades es controlando los ectoparásitos (Potter y Macintire, 2010).

\section{Literatura Citada}

1. Acevedo SP, Ramírez-Castrillón M, Restrepo LG 2009. Uveítis y glaucoma asociados a infección por Hepatozoon canis: reporte de un caso. Rev Colom Cienc Pec 22: 287-295.

2. Adagio LM, Miguel MC, Meder AR, Rio FJ, Gimenez M, Hierro JL, Mariani E, et al. 2014. Hepatozoonosis canina. Primeros 4 casos documentados en la ciudad de General Pico-Provincia de La Pampa-Argentina. Cienc Vet 16: 9-22. 
3. Allen KE, Li Y, Kaltenboeck B, Johnson EM, Reichard MV, Panciera RJ, Little SE. 2008. Diversity of Hepatozoon species in naturally infected dogs in the southern United States. Vet Parasitol 154: 220-225. doi: 10.1016/ j.vetpar.2008.03.027

4. Arcila Vh, Castellanos V, Díaz S, Sánchez M. 2005. Hepatozoon Canis en Colombia. Spei Domus 1: 41-45.

5. Baneth G, Samish M, Alekseev E, Aroch I, Shkap V. 2001. Transmission of Hepatozoon canis to dogs by naturally-fed or percutaneously-injected Rhipicephalus sanguineus ticks. J Parasitol 87: 606-611. doi: 10.1645/00223395(2001)087[0606:TOHCTD]2.0.CO;2

6. Baneth G, Mathew JS, Shkap V, Macintire DK, Barta JR, Ewing SA. 2003. Canine hepatozoonosis: two disease syndromes caused by separate Hepatozoon spp. Trends Parasitol 19: 27-31. doi: 10.1016/S1471-4922(02)00016-8

7. Baneth G, Samish M, Shkap V. 2007. Life cycle of Hepatozoon canis (Apicom-plexa: Adeleorina: Hepatozoidae) in the tick Rhipicephalus sanguineus and domestic dog (Canis familiaris). J Parasitol 93: 283-299. doi: 10.1645/GE-494R.1

8. Baneth G, Sheiner A, Eyal O, Hahn S, Beaufils JP, Anug Y, Talmi-Frank D. 2013. Redescription of Hepatozoon felis (Apicomplexa: Hepatozoidae) based on phylogenetic analysis, tissue and blood form morphology, and possible transplacental transmission. Parasite Vector 6: 102. doi: 10.1186/1756-33056-102

9. Castellanos VT. 2008. Hepatozoonosis canina, enfermedad emergente en Colombia. Spei Domus 12: 7-9.

10. De Fonseca T, Araújo E, dos Reis Lima LT, da Silva MG, Fonseca A, Costa S, Neto JB. 2016. Infecção por Hepatozoon sp em canino doméstico: Relato de caso. PUBVET 11: 207-312. doi: 10.22256/pubvet.v11n3.272-275
11. de Tommasi AS, Giannelli A, de Caprariis D, Ramos RA, de Paola G, Crescenzo G, et al. 2014. Failure of imidocarb dipropionate and toltrazuril/ emodepside plus clindamycin in treating Hepatozoon canis infection. Vet Parasitol 200: 242-245. doi: 10.1016/ j.vetpar.2013.12.013

12. Eljadar MSM, Singla LD, Mustafa RAA, Uppal SK. 2013. Morphometric variations in gametocytes of Hepatozoon canis from naturally infected dogs. $\mathrm{J}$ Parasitic Dis 37: 143-147. doi: 10.1007/ s12639-012-0149-5

13. Freeman L, Becvarova I, Cave N, Mac-Kay C, Nguyen P, Rama B, Takeshima R. 2011. Guía para la evaluación Nutricional. Clin Vet Peq Anim 31:91-102.

14. Gavazza A, Bizzeti M, Papini R. 2003. Observations on dogs found naturally infected with Hepatozoon canis in Italy. Rev Med Vet-Toulouse 154: 565-571.

15. Giannelli A, Lia RP, Annoscia G, Buonavoglia C, Lorusso E, DantasTorres F, et al. 2017. Rhipicephalus turanicus, a new vector of Hepatozoon canis. Parasitology 144: 730-737. doi: 10.1017/S003118201600250

16. Gómez E, Valle D, Toledo J, Simoni $Z$, Díaz A, Henriquez A, Nieves M. 2015. First discovery of Hepatozoon and other hemotropics in canine domestic of the Sucre Municipality, Sucre state, Venezuela. Bol Malar Salud Ambi 55: 94-104.

17. Greene CE. 2012. Infectious diseases of the dog and cat. $4^{\text {th }}$ ed. Missouri, USA: Elsevier Saunders. $1376 \mathrm{p}$.

18. Guendulain C, González G, Babini S, Caffaratti M, González P, Bessone A, Soler E, Tissera MC. 2017. Evaluación de la eficacia de algunos fármacos para el tratamiento de la hepatozoonosis canina. Analecta Vet 37(1). doi: 10.24215/ $15142590 \mathrm{e} 002$

19. Harmelin A, Dubey JP, Yakobson B, Nyska A, Orgad U. 1992. Concurrent Hepatozoon canis and Toxoplasma gondii infections in a dog. Vet Parasitol 43: 131-136. doi: 10.1016/03044017(92)90055-E 
20. Headley SA, Ribeiro EA, dos Santos GJ, Bettini CM, Júnior EM. 2005. Canine hypertrophic osteopathy associated with extra-thoracic lesions. Cienc Rural 35: 941-944. doi: 10.1590/ S0103-84782005000400033

21. Hervás J, Carrasco L, GómezVillamandos JC, Méndez, A, Sierra $M A .1995$. Acute fatal hepatozoonosis in a puppy: histopathological and ultrastructural study. Vet Rec 137: 518519. doi: 10.1136/vr.137.20.518

22. Iveli S, Casas L, Machuca M, Eiras DF, Del Amo A. 2015. Poliartritis asociada a hepatozoonosis canina: descripción de un caso. Analecta Vet 35: 25-29.

23. Lasta C, Santos A, Mello F, Lacerda L, Messick, J, Díaz González F. 2009. Infecção por Hepatozoon canis em canino doméstico na região Sul do Brasil confirmada por técnicas moleculares. Ciênc Rural 39: 2135-2140. doi: 10.1590/ S0103-84782009005000160

24. Maia JP, Crottini A, Harris DJ. 2014. Microscopic and molecular characterization of Hepatozoon domerguei (Apicomplexa) and Foleyella furcata (Nematoda) in wild endemic reptiles from Madagascar. Parasite 21: 1-14. doi: 10.1051/parasite/2014046

25. Mateus AA, Cala FA, Vargas G, Arcila VH, Castellanos V. 2007. Reporte de casos clínicos con Hepatozoon canis en el centro médico quirúrgico veterinario de la Universidad Cooperativa de Colombia. REDVET 8(5). [Internet]. Disponible en: http://www.veterinaria.org/ revistas/redvet/n050507/050705.pdf

26. Meneses A, Alvarado G, Runnebaum M, Herrera M, Gutiérrez-Espeleta G, Chaves A. 2016. Primer reporte de Hepatozoon procyonis en Mapaches de Costa Rica. Rev Cienc Vet 34: 5154. doi: 10.15359/rcv.34-1.4

27. Merino S, Martínez J, Masello JF, Bedolla Y, Quillfeldt P. 2014. First molecular characterization of a Hepatozoon species (Apicomplexa: Hepatozoidae) infecting birds and description of a new species infecting storm petrels (Aves: Hydrobatidae). J Parasitol 100: 338-343. doi: 10.1645/13325.1

28. Mejía C, Acevedo C. 2016. Diagnóstico histopatológico de esplenitis no supurativa en un canino producida por Hepatozoon spp. Un reporte de caso. Tesis de Especialización. Colombia: Univ. de Antioquia. $21 \mathrm{p}$.

29. Pasa S, Voyvoda H, Karagenc T, Atasoy A, Gazyagci S. 2011. Failure of combination therapy with imidocarb dipropionate and toltrazuril to clear Hepatozoon canis infection in dogs. Parasitol Res 109: 919-926. doi: 10.1007/ s00436-011-2334-3

30. Pedrozo R, Quintana G, Bazán A, Florentín M. 2010. Valores hematológicos de referencia en caninos adultos aparentemente sanos, que concurren a una clínica privada de Asunción. Mem Inst Investig Cienc Salud 8(2): 5-13.

31. Potter TM, Macintire DK. 2010. Hepatozoon americanum: an emerging disease in the south central/southeastern United States. J Vet Emerg Crit Care 20: 70-76. doi: 10.1111/j.14764431.2009.00508.x

32. Ramos CAM, Babo VJ, Pedroso TC, Souza AF, Araújo FE, Cleveland HPK. 2015. Molecular identification of Hepatozoon canis in dogs from Campo Grande, Mato Grosso do Sul, Brazil. Braz. J Vet Parasitol 24: 247-250. doi: 10.1590/S1984-29612015019

33. Restrepo CC. 2013. La ayuda diagnóstica es importante: caso de Hepatozoon spp. Biosalud 12: 121-126.

34. Rioux JA, Golvan YJ, Honin R. 1964. Mixed Hepatozoon canis and Leishmania canis infection in a dog in the Sets area, France. Ann Parasit Hum Comp 39: 131-135.

35. Ruiz MF, Zimmermann RN. 2013. Hallazgo de hepatozoon canis en caninos (canis familiaris) en la ciudad de esperanza, Santa Fe (Argentina). FAVE Cienc Vet 12: 15-20. doi: 10.14409/ favecv.v12i1/2.4542 
36. Smith TG. 1996. The genus Hepatozoon (Apicomplexa: Adeleina). J Parasitol 82: 565-585. doi: 10.2307/ 3283781

37. Sumrandee C, Baimai V, Trinachartvanit W, Ahantarig, A. 2015. Hepatozoon and Theileria species detected in ticks collected from mammals and snakes in Thailand. Ticks Tick-Borne Dis 6: 309-315. doi: 10.1016/ j.ttbdis.2015.02.003
38. Tintel M. 2016. Reporte de cinco casos clínicos de hepatozoonosis en caninos de Paraguay. REDVET 17(9). [Internet]. Disponible en: http://www. veterinaria.org/revistas/redvet/n090916/ 091611.pdf

39. Van As J, Davies AJ, Smit NJ. 2015. Life cycle of Hepatozoon affluomaloti sp. n. (Apicomplexa: Haemogregarinidae) in crag lizards (Sauria: Cordylidae) and in culicine mosquitoes from South Africa. Folia Parasit 62: 1-9. doi: 10.14411/fp.2015.008 